\title{
Presencia de marcadores de las vías de activación de los macrófagos en periodontitis crónica
}

\section{Presence of markers of activation pathways of macrophages in chronic periodontitis}

\author{
Navarrete $\mathrm{M}^{1}$, García-Sesnich $\mathrm{J}^{2}$, Dutzan $\mathrm{N}^{3}$, Henríquez L4 , Puente $\mathrm{J}^{5}$, Gamonal $\mathrm{J}^{6}$
}

\begin{abstract}
RESUMEN
La periodontitis crónica es una patología infecciosa, causada por un complejo de especies bacterianas, que afecta principalmente los tejidos de inserción de los dientes. La respuesta inmune-inflamatoria producida se caracteriza por la presencia de un infiltrado inflamatorio, en el cual los macrófagos representan entre 5 al $30 \%$. Es sabido que los macrófagos se activan mediante dos vías: Clásica y Alterna, caracterizadas por la presencia de marcadores indirectos: IFN-y e IL-6 para la vía clásica e IL-4 para la vía alterna, ampliamente abordados. Recientemente, se ha descrito a la subunidad A del factor XIII de la coagulación (FXIII-A) como un buen marcador de la vía alterna. El objetivo de este estudio consiste en determinar la presencia de IFN-y, IL-6, FXIII-A e IL-4 como marcadores de las vías de activación de los macrófagos, en pacientes con periodontitis crónica. Para tal efecto, se realizó inmunohistoquímica y Western-Blot para los cuatro marcadores junto a CD-68, marcador de macrófagos, en 18 biopsias de tejido periodontal sano y 18 con periodontitis crónica. Se detectó la presencia de IFN- $\gamma$, IL-6, IL-4 y FXIII-A junto a CD68+, en todas las muestras de pacientes sanos y con periodontitis. Los resultados obtenidos sugieren que al estar presente IFN-y, IL-6, IL-4 y FXIII-A, los macrófagos se activarían a través de ambas vías, lo cual, produciría una respuesta tanto proinflamatoria (Th1) como antinflamatoria (Th2). Son necesarios más estudios para determinar si existe una vía preferencial de activación.
\end{abstract}

Rev. Clin. Periodoncia Implantol. Rehabil. Oral Vol. 5(3); 131-134, 2012.

Palabras clave: Factor XIII-A, macrófagos, periodontitis.

\section{ABSTRACT}

Periodontitis is a chronic infectious disease caused by a bacterial species complex, which affects mainly the insertion tissues of the teeth. The immuneinflammatory response produced is characterized by an inflammatory infiltrate in which macrophages represent between 5 to $30 \%$. It is known and has been widely discussed that macrophages are activated in two ways: Classical and Alterna, characterized by the presence of indirect markers: IFN- $\gamma$ and IL-6 for the classical pathway and IL-4 for the alternative pathway. Recently the subunit A of the clotting factor XIII (FXIII-A) has been described as a good marker of the alternative pathway. The objective of this study is to determine the presence of IFN-y, IL-6, IL-4 and FXIII-A as markers of the macrophage activation pathways in patients with chronic periodontitis. To this end, we performed immunohistochemistry and Western blot for the four markers with CD68 macrophage marker, in 18 healthy periodontal tissue biopsies and 18 with chronic periodontitis. We detected the presence of IFN- $y$, IL-6, IL-4 and FXIII-A with CD68 +, in all samples of healthy patients and periodontitis. The results suggest that when present, IFN- $y$, IL-6, IL-4 and FXIII-A, activate macrophages through both routes, which would produce a proinflammatory response (Th1) as antiinflammatory (Th2). Further studies are necessary to determine whether there is a preferential pathway activation.

Rev. Clin. Periodoncia Implantol. Rehabil. Oral Vol. 5(3); 131-134, 2012.

Key words: Factor XIII-A, macrophages, periodontitis.

\section{INTRODUCCIÓN}

Losmacrófagos son células mononucleadas que se caracterizan por su capacidad de fagocitar y degradar material particulado. Se sabe que representan cerca de 5 a $30 \%$ del infiltrado inflamatorio presente en las lesiones periodontales ${ }^{(1)}$. En periodontitis, estas células participan tanto en la respuesta inmunológica inespecífica frente al desafío microbiano, fagocitando microorganismos y secretando citoquinas inflamatorias, como en la específica, donde actúan principalmente como células presentadoras de antígenos (APCs) ${ }^{(2)}$. Para poder ejercer esta variedad de funciones, el macrófago se activa mediante dos vías. La vía clásica inducida por citoquinas asociadas a respuesta Th1 como IFN-y e IL-6 principalmente y la vía alterna por citoquinas asociadas a Th2 como IL-4. Estas citoquinas también actúan como marcadores para sus respectivas vías. Dentro de los marcadores descritos para los macrófagos, CD68 es uno de los más utilizados ${ }^{(3)}$ y para reconocer la(s) vía(s) de activación de éstas células en pacientes con periodontitis, también se necesitan marcadores de los cuales IFN-y, IL-6 (vía clásica ${ }^{(4)}$ ) e IL-4 (vía alterna $\left.{ }^{(5)}\right)$,

1. Cirujano Dentista. Diplomada en Periodoncia, Facultad de Odontología, Universidad de Valparaíso. Magíster en Cs. Odontológicas, Mención Periodontología. Profesor Asistente, Facultad de Odontología, Cátedra Periodoncia. Universidad Andrés Bello. Viña del Mar, Chile.

2. Bioquímico. Laboratorio de Biología Periodontal. Facultad de Odontología, Universidad de Chile. Chile.

3. Cirujano Dentista. Especialista en Periodoncia, Universidad de Chile. Magíster en Cs. Odontológicas, Mención Periodontología. Profesor Asistente, Área de Periodoncia, Laboratorio de Biología Periodontal, Departamento de Odontología Conservadora. Facultad de Odontología, Universidad de Chile. Chile.

4. Técnico Paramédico. Laboratorio de Biología Periodontal. Facultad de Odontología, Universidad de Chile. Chile.

5. Bioquímico. Doctor en Bioquímica, Departamento De Bioquímica y Biología Molecular. Facultad de Ciencias Químicas y Farmacéutica, Universidad de Chile. Chile.

6. Cirujano Dentista. Especialista en Periodoncia. Magíster y Doctor en Cs. Odontológicas. Laboratorio de Biología Periodontal, Departamento de Odontología Conservadora. Facultad de Odontología, Universidad de Chile. Chile.

Correspondencia autor: Mariely Navarrete Riffo. marielynr@gmail.com. Laboratorio de Biología Periodontal. Facultad de Odontología, Universidad de Chile. Sergio Livingstone Pohlhammer 943. Independencia. Santiago, Chile. Financiamiento: Proyecto FONDECYT 1090046. Trabajo recibido el 27/08/2012. Aprobado para su publicación el 26/10/2012. 
han sido ampliamente abordados. Estudios recientes han descrito al Factor XIII (FXIII) como un buen marcador para la vía alterna. EI FXIII es un factor de la coagulación estabilizador de la fibrina6, presente en la circulación en forma tetramérica con 2 unidades A catalíticas y 2 B inhibidoras y la participación de su subunidad A (FXIII-A) (7) no solo se limita a la hemostasia, sino también está presente en la cicatrización de las heridas y mantención del embarazo ${ }^{(8,9)}$. En estudios anteriores, se establece claramente el rol del FXIII-A como marcador de la vía de activación alterna del macrófago, ya que hay un incremento ostensible en la expresión de ARNm de FXIII-A y de la proteína propiamente tal, en macrófagos activados por esta vía ${ }^{(10)}$. Además, se demostró que la ausencia de FXIII-A en la célula indicaba que su activación correspondía a la vía clásica y que el aumento en la expresión génica de FXIII-A producido por IL-4, podía ser inhibido al exponer las células activadas frente a estímulos de la vía de activación clásica. Teniendo en cuenta la diversidad y relevancia de las funciones efectoras proinflamatorias desarrolladas por los macrófagos activados por la vía clásica, junto al rol preponderante de la vía alterna en la regulación de la inflamación y su participación en los procesos reparativos, es importante determinar si los macrófagos presentes en pacientes con periodontitis, se encuentran activados por una o ambas vías.

El objetivo de este estudio consiste en determinar la presencia de IFN-y, IL-6, FXIII-A e IL-4, como marcadores de las vías de activación de los macrófagos, en pacientes con periodontitis crónica.

\section{MATERIALES Y MÉTODOS}

De los sujetos que solicitan atención en la Facultad de Odontología, Universidad de Chile (Santiago, Chile), se seleccionaron 18 pacientes con periodontitis crónica y 18 sujetos sanos periodontalmente que cumplían con todos los criterios de inclusión y manifestaron su intención de participar voluntariamente, firmando un consentimiento informado. El protocolo y diseño de este estudio fue sometido a aprobación por el Comité de Ética de la Facultad de Odontología, Universidad de Chile y por el Fondo Nacional de Desarrollo Científico y Tecnológico (FONDECYT).

Los criterios de inclusión para los pacientes con periodontitis crónica fueron los siguientes: Pacientes $\geq 35$ años, con un número $>14$ dientes naturales presentes (por lo menos 10 dientes posteriores, excluyendo terceros molares), con enfermedad periodontal crónica moderada o avanzada(11), diagnosticándose esta patología en aquellos sujetos que presentaban al menos 4 sitios de profundidad al sondaje mayor a $5 \mathrm{~mm}, 6$ sitios con pérdida de inserción mayor a $3 \mathrm{~mm}$ y evidencia radiográfica de destrucción ósea alveolar. En el grupo control se incluyeron individuos periodontalmente sanos, sometidos a cirugía de alargamiento coronario o exodoncia de terceros molares.

Los criterios de exclusión para ambos grupos, se relatan a continuación: Historia de tratamiento periodontal previo, medicación antibiótica 3 a 6 meses previos al estudio, fumadores, antecedentes de enfermedad autoinmune, infecciosa y/o inflamatoria en el transcurso de los últimos 6 meses, afecciones sistémicas que pudieran afectar el desarrollo de la enfermedad periodontal y embarazo.

Para obtener las muestras de tejido gingival, según protocolo de Ramfjord y Nissle (1974) ${ }^{(12)}$, se realizó una biopsia a aquellos sitios con profundidad al sondaje mayor o igual a $5 \mathrm{~mm}$ en los pacientes o el sitio a intervenir en los sujetos sanos. Previa anestesia local del sitio seleccionado, retiro de placa supra gingival y aislamiento relativo con tórulas de algodón, con una hoja $\mathrm{N}^{\circ} 15$ se realizó una incisión a 1-2 mm desde el margen gingival, dirigida hacia la cresta alveolar, siguiendo el eje mayor del diente, de modo que la biopsia contenga epitelio y tejido conectivo.

Una vez tomada la biopsia, se sumergió en $5 \mathrm{ml}$ de medio de cultivo RPMI 1640 estéril, suplementado con $50 \mathrm{UI} / \mathrm{ml}$ de penicilina, 50 $\mu \mathrm{g} / \mathrm{ml}$ de estreptomicina y L-glutamina $200 \mathrm{mM}$ (SIGMA Chemical Co., St. Louis, MO, USA) y se trasportó inmediatamente en hielo al Laboratorio de Biología Periodontal de la Facultad de Odontología, Universidad de Chile, donde se lavaron 3 veces con $\mathrm{NaCL}$ al $0.9 \%$, se pesaron en balanza analítica y se guardaron en seco a $-80^{\circ} \mathrm{C}$, hasta su procesamiento.

Para realizar inmunohistoquímica, las biopsias de tejido gingival son fijadas en formalina buffer $4 \%$, deshidratadas e incluidas en parafina para su almacenamiento. Luego, obtienen corte de $4 \mu \mathrm{m}$, los cuales se desparafinan y rehidratan mediante protocolo estándar. Se utilizaron anticuerpo primario mono clonales para: IL-6 (monoclonal anti-human IL-6 de R y D Systems numero de catálogo MAB2061) en una dilución de 1:50; anti-human FXIII-A, (Epitomics, número de catálogo 2559-1) en una dilución de 1:100; antihuman IL-4 (Epitomics, número de catálogo 2177-1) en una dilución de 1:100; antihuman INF-Y (Epitomics, número de catálogo 1833-1) en una dilución de 1:25 y Mouse anti-CD68, Clone KP1 (Invitrogen, numero de catálogo 08-0125) prediluído en una dilución de 1:50.

El procesamiento de los tejidos comienza con el bloqueo de la peroxidasa y desemascaramiento antigénico con Buffer Citrato 0.01 $\mathrm{M} \mathrm{pH}$ 6. Luego se procedió a marcar con el anticuerpo seleccionado, el cual se incubó durante la noche a $4^{\circ} \mathrm{C}$, excepto anti-CD68 que se incubó una hora. Para la tinción se utilizó un método estándar de avidina-biotina peroxidasa (Vectastain, Elite ABCKit, Vector; Vector Laboratories Inc., Burlingame, CA, USA) según las indicaciones del fabricante. Se revela con el kit DAB para peroxidasa (Impact DAB Kit for Peroxidase; Vector Laboratories) como cromógeno y contratinción nuclear hematoxilina. En cada procedimiento de tinción se realizó un control negativo sin los anticuerpos primarios. Biopsias de amígdalas humanas se utilizaron como controles positivos. Finalmente, las muestras fueron deshidratadas y montadas con medio de montaje hidrofóbico (Flotex®, Lerner Laboratories, Pittsburgh, PA, USA) y una vez realizado el marcaje inmunohistoquímico, los cortes estudiados fueron examinados con un microscopio óptico (Zeiss, Axiostar Plus ${ }^{\circledR}$ ) y se les tomó fotografías a las áreas elegidas con una cámara de fotos (Power Shot A640, Cannon) y se digitalizaron mediante un sofware (Carl Zeiss Vision Axiovisionrel).

Para realizar la detección de FXIII-A, IL-4, IFN- $\gamma$, IL-6 y de la proteína globular Actina, utilizada como control de carga mediante Western Blot (WB), de las biopsias guardadas en seco a $-80^{\circ} \mathrm{C}$ se prepararon homogenizados mediante un homogenizador mecánico y su dilución a $2 \mathrm{ml}$ con cloruro de sodio al $0.9 \%$. La medición de proteínas totales se realizó mediante el método del ácido bisciconitico (Micro BCA Protein Assay Reagent Kit, Pierce Biotechnology, Rockford, USA), siguiendo las indicaciones del proveedor. Luego, se prepararon geles de trisglicina al $15 \%$ cargando muestras de individuos sanos y enfermos, más control de peso molecular (Benchmark Prestained Protein Ladder, Cat Nº107478-010, Invitrogen). Se corre el gel, posteriormente se transfiere a una membrana de PDVF la cual bloqueada con suero bovino fetal al 3\% (BSA-50, Rockland Inc.) y se incuba con el anticuerpo primario de: IL-6 (monoclonal anti-human IL-6 de R y D Systems número de catálogo MAB2061), en una dilución de 1:250; anti-human FXIII-A (Epitomics, número de catálogo 2559-1), en una dilución de 1.10000; antihuman IL-4 (Epitomics, número de catálogo 2177-1), en una dilución de 1:1000; antihuman INF-Y (Epitomics, número de catálogo 1833-1), en una dilución de 1:2000 y Actina: Beta Actin antibody (Abcam número de catálogo 8226) en una dilución de 1:5000, junto a la membrana a $37^{\circ} \mathrm{C}$, toda la noche. Al día siguiente se le adiciona el anticuerpo secundario GAMPO: Stabilized Goat Anti-Mouse IgG $(\mathrm{H}+\mathrm{L})$, Peroxidase Conjugated número de catálogo 32430) en una dilución 1:10000 para Actina e IL-6 y GARPO: goat anti-rabbit HRP-conjugated antibody ( Pierce, número de catálogo 1858415) en una dilución 1:10000 para IL-4, FXIII-A e INF- $y$, a $37^{\circ} \mathrm{C}$ por una hora, y se revela mediante quimioluminiscencia (SuperSignal West Femto, Pierce Biotechnology, Rockford, USA) en placas radiográficas.

\section{RESULTADOS}

Las características clínicas de los sujetos incluídos en el estudio se detallan en la Tabla 1. Se aprecian diferencias estadísticamente significativas en todos los parámetros clínicos al comparar ambos grupos, por lo cual, podemos inferir que en los sitios de pacientes con periodontitis tienen mayor destrucción que los sitios de sujetos sin enfermedad.

Se detectó la presencia de macrófagos mediante su marcador de superficie CD-68, en el infiltrado inflamatorio de todas las muestras de pacientes sanos y con periodontitis, siendo más evidente en biopsias de pacientes con periodontitis (Figura 1). Se observan también un gran número de células cuya morfología es compatible con linfocitos (en azul).

La presencia tanto de IFN-y como IL-6 fue detectada en todas las muestras de tejido periodontal, pudiéndose distinguir pequeños focos al interior del infiltrado inflamatorio en los tejidos sanos, en contraste con las biopsias de pacientes con periodontitis donde se observa un infiltrado inflamatorio mucho más denso, lo cual, caracteriza a los tejidos periodontales enfermos Por lo tanto, la presencia los marcadores de la vía de activación clásica de los macrófagos IFN-y (Figura 2A). e IL-6 
(Figura 2B), es mucho más evidente en las muestras de pacientes con periodontitis, que en aquellas de pacientes sanos periodontalmente.

Por otra parte, la presencia de un denso infiltrado inflamatorio y de los marcadores IL-4 (Figura 3A) y FXIII-A (Figura 3B), en las muestras de tejido periodontal enfermo, fue notoriamente más marcada respecto a las biopsias de pacientes periodontalmente sanos, donde se pudo distinguir pequeños focos al interior del infiltrado inflamatorio (Figura 3 , flechas negras).Por lo tanto, la presencia los marcadores FXIII-4 e IL-4 es mucho más notoria en las muestras de pacientes con periodontitis, que en aquellas de pacientes sanos periodontalmente.

En resumen, se demuestra una marcada presencia de IFN- $\gamma$, IL-6, FXIII-A e IL-4, marcadores de la vía de activación clásica y alterna de los macrófagos respectivamente, en las biopsias de pacientes con periodontitis versus pacientes periodontalmente sanos.

Para realizar WB se tomaron 12 muestras de tejido gingival sano y 12 tejido enfermo. En todos los ensayos realizados se detectó la presencia de los cuatro marcadores de las vías de activación de los macrófagos (IFN- $\mathrm{y}, \mathrm{IL}-6$, FXIII-A e IL-4), en todas las muestras de tejido gingival. Como una forma de estandarizar más aun los resultados, se tomaron otras 3 muestras de pacientes periodontalmente sanos y 3 muestras de pacientes con periodontitis y se realizó WB para cada uno de los marcadores. Además, con estas mismas muestras se hizo un nuevo WB para Actina, la cual, se utilizó como control de carga.

La presencia de IFN- $\gamma$ fue detectada en todas las muestras de tejido gingival, a través de bandas ubicadas en un peso molecular de 26 kDa (Figura 4), cuya intensidad y tamaño no presenta variación entre las muestras de pacientes sanos y enfermos.

Tabla 1. Características clínicas de los pacientes periodontalmente sanos y con periodontitis (promedio \pm SD).

\begin{tabular}{|l|c|c|}
\hline & $\begin{array}{c}\text { Sanos } \\
(\mathrm{N}=18)\end{array}$ & $\begin{array}{c}\text { Periodontitis } \\
(\mathrm{N}=18)\end{array}$ \\
\hline Edad (promedio $\pm \mathrm{SD})$ & $36.38 \pm 3.20 £$ & $47.30+4.47 £$ \\
\hline$\%$ Mujeres & $50 ¥$ & $84 ¥$ \\
\hline Profundidad de sondaje (mm, promedio $\pm \mathrm{SD})$ & $1.77 \pm 0.63^{*}$ & $3.06 \pm 0.65^{*}$ \\
\hline Nivel de inserción clínica (mm, promedio $\pm \mathrm{SD})$ & $0.77 \pm 0.89 \dagger$ & $3.43 \pm 0.59 \dagger$ \\
\hline \% sitios con placa & $22.5 \S$ & $91.63 \S$ \\
\hline$\%$ sitios con sangramiento al sondaje & $0.02 \ddagger$ & $43.5 \ddagger$ \\
\hline
\end{tabular}

$£$ Promedio de la edad de los pacientes sanos versus pacientes con periodontitis: $\mathrm{P}=0.0001$ $¥$ Porcentaje mujeres sanas versus mujeres con periodontitis: $P=0.031$.

* Promedio Profundidad de Sondaje de pacientes sanos versus pacientes con periodontitis: $P=0.001$ † Promedio Nivel de Inserción Clínica de pacientes sanos versus pacientes con periodontitis: $P=0.0001$. $\S$ Porcentaje sitios con placa de pacientes sanos versus pacientes con periodontitis: $\mathrm{P}=0.0001$. $\ddagger$ Porcentaje sitios con sangramiento al sondaje de pacientes sanos versus pacientes con periodontitis: $P=0.0007$. (Test t no pareado y test chi cuadrado).

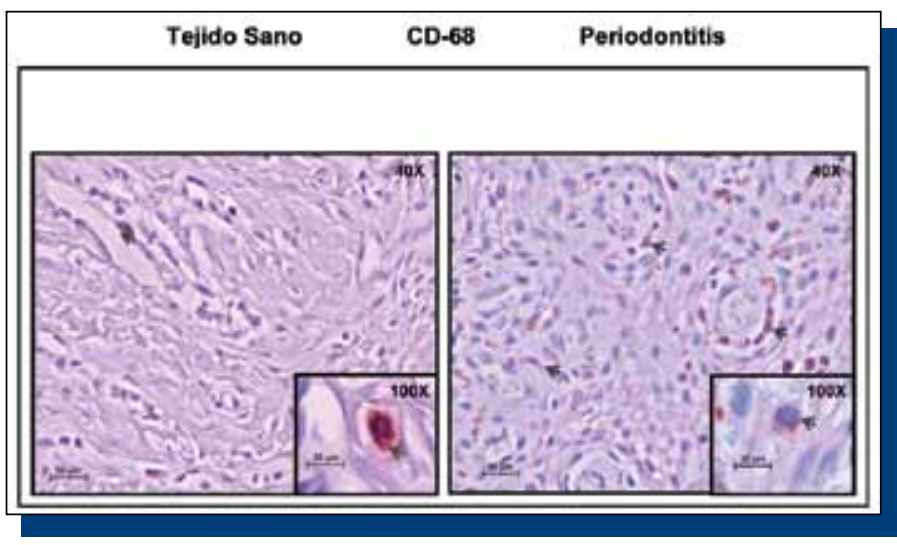

Figura 1. Inmunohistoquímica de biopsias de tejido periodontal marcadas con anticuerpo CD-68 (flechas negras, con aumentos de 40X y 100X). Se detectó la presencia de macrófagos en un denso infiltrado inflamatorio de pacientes periodontalmente sanos $(n=10)$ y con periodontitis $(n=10)$, destacándose la presencia de células (en azul) cuya morfología es compatible con linfocitos.

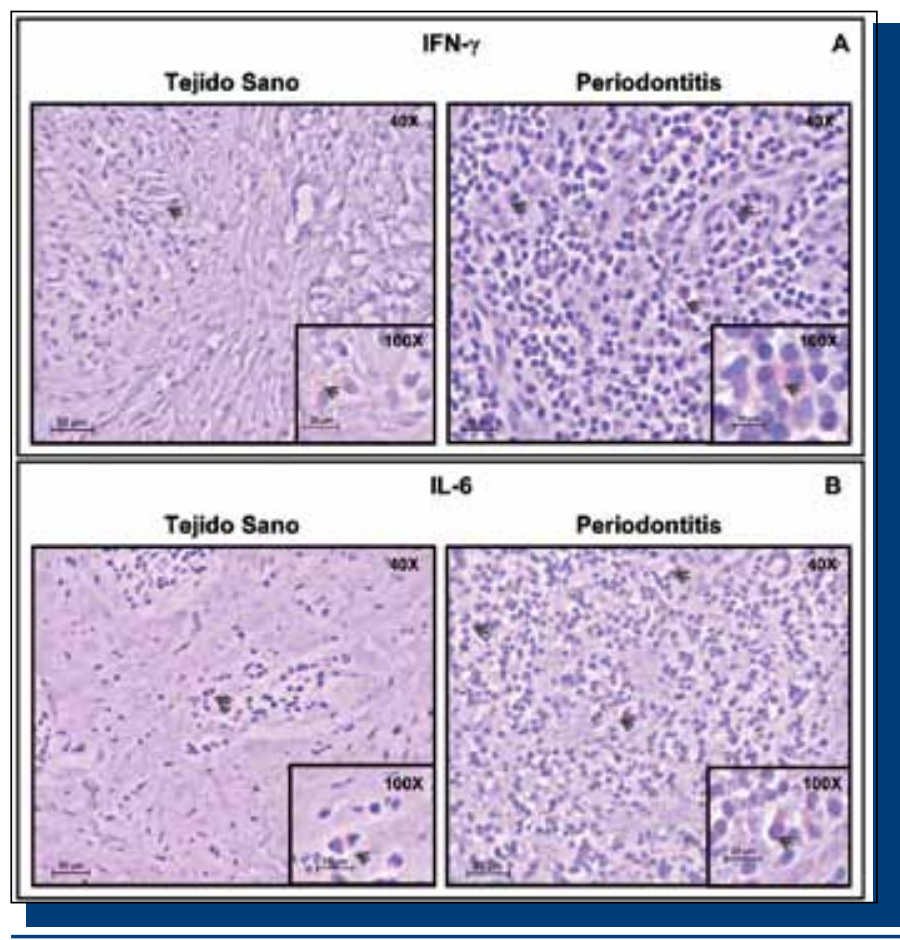

Figura 2. Inmunohistoquímica marcadores de la vía de activación clásica de los macrófagos. Biopsias de tejido periodontal marcadas con anticuerpo para A: IFN- $\gamma$ y B: IL-6. Se distingue la presencia de ambos marcadores de la vía clásica (flechas negras), con aumentos de 40X y 100X. A diferencia de las muestras de tejido sano $(n=10)$ donde se observan pequeños focos inflamatorios, se detecta un denso infiltrado inflamatorio en las muestras de pacientes con periodontitis $(n=10)$, donde el número de células marcadas por IFN- $\gamma$ e IL-6, es mucho mayor.
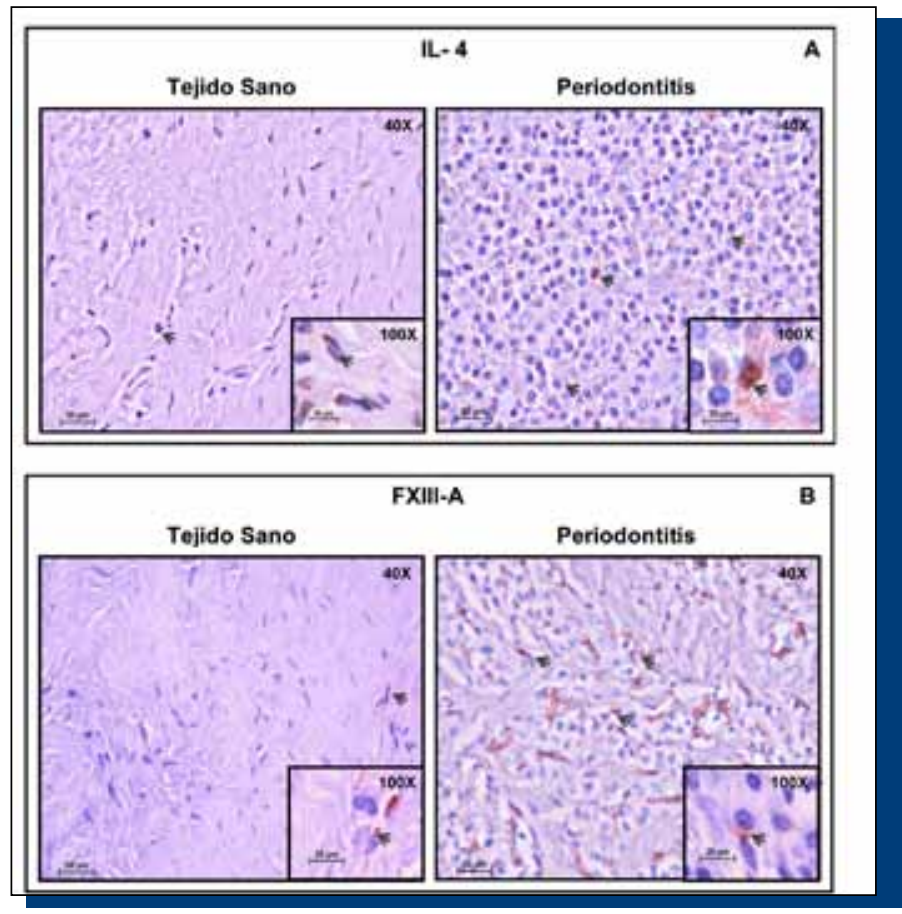

Figura 3. Inmunohistoquímica de la vía de activación alterna de los macrófagos Biopsias de tejido periodontal marcadas con anticuerpo para A: IL-4 y B: FXIII-A Se distingue la presencia de ambos marcadores de la vía clásica (flechas negras), con aumentos de 40X y 100X. A diferencia de las muestras de tejido sano $(n=10)$ donde se observan pequeños focos inflamatorios, se detecta un denso infiltrado inflamatorio en las muestras de pacientes con periodontitis $(n=10)$, donde el número de células marcadas por FXIII-A e IL-4, es mucho mayor. Esta diferencia es mucho más evidente en las biopsias donde se utilizaron marcadores de la vía alterna de activación de los macrófagos, respecto a los de la vía clásica.

Para el marcador IL-6 se observan bandas de $17 \mathrm{kDa}$, muy marcadas y de mayor tamaño respecto a IFN-y, en todas las muestras de pacientes periodontalmente sanos y en periodontitis (Figura 4). 
La presencia de FXIII-A fue detectada en todas las muestras de tejido gingival, visualizándose bandas de $80 \mathrm{kDa}$ (Figura 4). En el WB de IL-4, la presencia del marcador se observó en todas las muestras de tejido gingival, a través de bandas ubicadas en un peso molecular de 14 kDa (Figura 4), cuya intensidad y tamaño no presenta variación entre las muestras de pacientes sanos y enfermos.

Comparativamente, las bandas correspondientes a IL-4 son de mayor tamaño e intensidad respecto a las de FXIII-A

Finalmente, se realizó WB para Actina, con las mismas muestras utilizadas anteriormente para los WB de IFN- $\gamma$, IL-6, FXIII-A e IL-4, observándose bandas cuyo peso molecular correspondía a 42 kDa (Figura 4).

En resumen, se detectó la presencia de los cuatro marcadores de las vías de activación de los macrófagos, en todas las muestras de tejido gingival, a través de la visualización de bandas cuyos pesos moleculares coincidían con los marcadores en estudio. En los WB de IFN-y, IL-6 e IL-4, el tamaño e intensidad de las bandas observadas es muy similar entre sujetos sanos y con periodontitis.

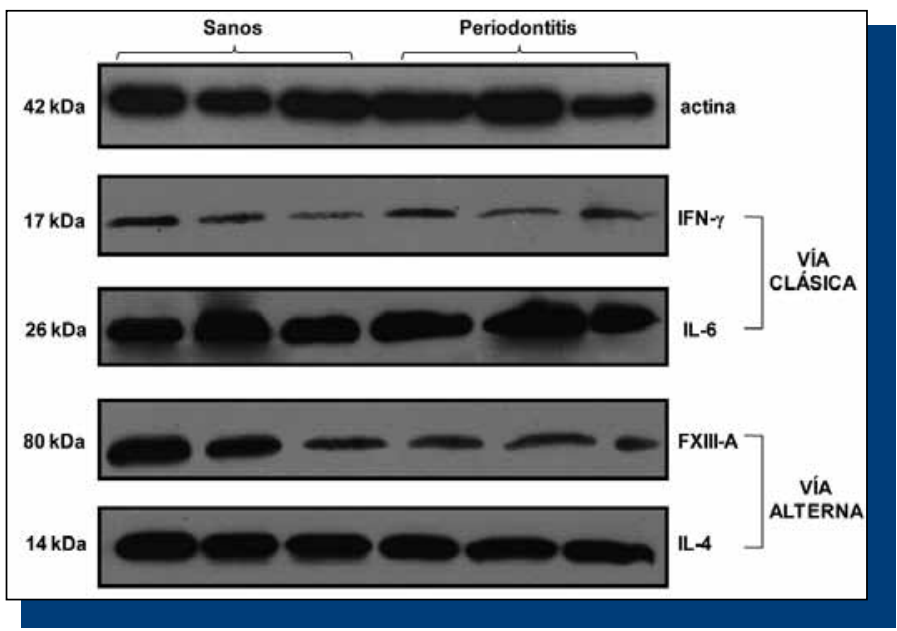

Figura 4. Western Blot de los marcadores de la vía clásica (IFN- $\gamma$ e IL-6) y alterna (FXIII-A e IL-4) de activación de los macrófagos y de Actina utilizada como control de carga. Se detectó la presencia de los cuatro marcadores y Actina, en todas las muestras de tejido al sano y enfermo, por visualización de bandas ubicadas en los pesos moleculares correspondientes a IFN- $\gamma$ e IL-6, FXIII-A, IL-4 y Actina. Se observan bandas de menor intensidad y tamaño en WB de IFN- $\gamma$ en comparación al resto de los marcadores y al control de carga Actina.

\section{DISCUSIÓN}

Los macrófagos son una de las poblaciones celulares más pleiotrópicas del sistema inmune. Ellos son efectores de la respuesta innata y están involucrados en el inicio y regulación de las respuestas adaptativas ${ }^{(13)}$.

Aunque la periodontitis ha sido extensamente estudiada, no existen aun datos suficientes para describir completamente el rol de los macrófagos en esta enfermedad ${ }^{(14)}$. En cuanto a su distribución en los tejidos gingivales, estas células se encuentran presentes en gran numero en los tejidos adyacentes al epitelio oral(14) y en relación a la presencia de los macrófagos en salud y enfermedad periodontal, un estudio semi cuantitativo demostró que los macrófagos se distribuyen de manera similar tanto en tejidos de pacientes con gingivitis como en tejidos de pacientes tratados periodontalmente ${ }^{(15)}$. En contraste a esto, en los resultados obtenidos por este estudio mediante inmunohistoquímica, se detectó la presencia de macrófagos mediante su marcador de superficie CD-68, en el infiltrado inflamatorio de todas las muestras de tejido gingival y la marca fue más evidente en biopsias de pacientes con periodontitis. Con respecto a la activación de los macrófagos, se realizó un estudio utilizando un modelo experimental de gingivitis, con el fin de analizar los cambios celulares durante un periodo de 33 días. Los resultados apuntaron a que los macrófagos son extremadamente reactivos frente a los distintos niveles de la inflamación ${ }^{(16)}$. Respecto a esto último, nuestro estudio demostró mediante IHQ y WB, la presencia de IFN-y, IL-6, FXIII-A e IL-4, marcadores de la vías de activación de los macrófagos, en todas las muestras de tejido. Sin embargo, mediante IHQ se obtuvo una marca mucho más intensa en las biopsias de pacientes con periodontitis versus pacientes periodontalmente sanos. Todo esto hace suponer que si bien ambas vías de activación están presentes en salud y enfermedad, es en esta última donde las vías clásica y alterna, promueven en mayor cuantía su patrón de respuesta. Entonces, si existe una mayor presencia de los cuatro marcadores en tejidos de pacientes con periodontitis, sumado a la alta reactividad de los macrófagos frente a distintos niveles de inflamación, lo cual, es característico de la periodontitis, el macrófago presente en enfermedad periodontal podría ser activado por ambas vías. El predominio de una sobre otra, si es que existe, va a depender de las necesidades del sitio enfermo, enmarcadas dentro de un tiempo y espacio particular.

Por todo esto, las vías de activación de estas células se han convertido en un tópico de gran impacto, debido a su determinante influencia. Si bien actualmente se habla de diferentes estados de activación, entre los que se encuentran el clásico y el alternativo, no está del todo claro cuáles son las propiedades funcionales y el repertorio molecular de cada uno de estos grupos de macrófagos.

Finalmente, los resultados de este estudio para los pacientes periodontalmente sanos, demuestran la presencia de IFN-y e IL-6, marcadores de la vía clásica y FXIII-A e IL-4, marcadores de la vía alterna, en muestras de tejido gingival, mediante WB e IHQ. Sin embargo, se observa por medio de esta última técnica, que la presencia de IFN- $y$, IL-6, FXIII-A e IL-4 sería menor en los tejidos sanos comparados con los tejidos de pacientes con periodontitis. Respecto a la presencia de IFN- $\gamma$, IL-6, IL-4 y FXIII-A en muestras de tejido gingival de pacientes con periodontitis, esta fue demostrada por WB e IHQ, lo cual, se manifiesta notoriamente por medio de esta última técnica en los tejidos enfermos.

Se sugiere realizar más estudios al respecto utilizando los marcadores anteriormente citados, en especial FXIII-A, cuantificando la expresión de estos últimos para poder compararlos entre si, en tejidos gingivales de pacientes sanos y con periodontitis, detectando posibles cambios en los niveles de los marcadores, lo cual, podría extrapolarse a cambios en las vías de activación del macrófago.

\section{CONFLICTOS DE INTERÉS}

Los autores declaran no tener conflictos de interés.

\section{REFERENCIAS BIBLIOGRÁFICAS}

1. Chomyszyn-Gajewska M, Czajkowska B, Blazewicz M, Pamula E, Ptak M. In vitro response of macrophages to a new carbon-polylactide composite for the treatment of periodontal diseases. Biomaterials, 2002; 23(2): 463-470.

2. Ali H, Haribabu B, Richardson RM, Snyderman R. Mechanisms of inflammation and leukocyte activation. Med Clin North Am, 1997; 81(1): 1-28.

3. Pulford KA, Rigney EM, Micklem KJ, Jones M, Stross WP, Gatter KC et al. KP1: A new monoclonal antibody that detects a monocyte/macrophage associated antigen in routinely processed tissue sections. J Clin Pathol, 1989; 42(4): 414-421.

4. Nathan C. Mechanisms and modulation of macrophage activation. Behring Inst Mitt, 1991; 88: 200-207.

5. Mosser DM. The many faces of macrophage activation. J Leukoc Biol, 2003; 73(2): 209-212.

6. Muszbek L, Adany R, Mikkola H. Novel aspects of blood coagulation factor XIII. I. Structure, distribution, activation, and function. Crit Rev Clin Lab Sci, 1996; 33(5): 357-421

7. Komaromi I, Bagoly Z, Muszbek L. Factor XIII: Novel structural and functional aspects. J Thromb Haemost, 2011; 9(1): 9-20.

8. Asahina T, Kobayashi T, Okada Y, Goto J, Terao T. Maternal blood coagulation factor XIII is associated with the development of cytotrophoblastic shell. Placenta 2000;21(4):388-93
9. Lorand L. Factor XIII and the clotting of fibrinogen: From basic research to medicine. J Thromb Haemost, 2005; 3(7): 1337-1348.

10. Torocsik D, Bardos H, Nagy L, Adany R. Identification of factor XIII-A as a marker of alternative macrophage activation. Cell Mol Life Sci, 2005; 62(18): 2132-2139.

11. Armitage GC. Periodontal diagnoses and classification of periodontal diseases. Periodontol 2000, 2004; 34: 9-21.

12. Ramfjord SP, Nissle RR. The modified widman flap. J Periodontol, 1974; 45(8): 601-607

13. Duque C. Activación alternativa del macrófago: La diversidad en las respuestas de una célula de la inmunidad. Inmunología, 2007; 26(2): 73-86.

14. Chapple CC, Srivastava M, Hunter N. Failure of macrophage activation in destructive periodontal disease. J Pathol, 1998; 186(3): 281-286.

15. Schlegel Gomez R, Langer P, Pelka M, von den Driesch P, Johannessen AC, Simon M Jr. Variational expression of functionally different macrophage markers (27E10, 25F9, RM3/1) in normal gingiva and inflammatory periodontal disease. $J$ Clin Periodontol, 1995; 22(5): 341-346.

16. Topoll HH, Zwadlo G, Lange DE, Sorg C. Phenotypic dynamics of macrophage subpopulations during human experimental gingivitis. J Periodontal Res, 1989; 24(2): 106-112. 